\title{
URLLC Packet Management for Packetized Predictive Control
}

\author{
Qi Wang*, Sha Xie*, Guodong Zhao ${ }^{\dagger}$, Lei Zhang ${ }^{\dagger}$, and Zhi Chen* \\ ${ }^{*}$ National Key Lab. on Communications, UESTC, Chengdu, China \\ ${ }^{\dagger}$ School of Engineering, University of Glasgow, Glasgow, UK
}

\begin{abstract}
Packetized predictive control (PPC) is an effective solution to ensure the robustness of the control system over unreliable wireless links. However, conventional wireless transmission methods in PPC suffer from either high wireless resource consumption or poor performance of real-time control due to the separately design of the two parts. To deal with the issue, we propose a communicationcontrol co-design approach to achieve good trade-off between real-time control performance and communication energy efficiency. Our results demonstrate the advantages of the communication-control co-design.
\end{abstract}

\section{INTRODUCTION}

Industrial automation, automatic driving and mobile medical services are identified as of paramount importance application scenarios for 5G-and-beyond [1] [3]. To fully support these applications, it is critical to provide ultra high-reliable and low latency communications (URLLC) [4]-[7], with the end-to-end latency requirement being as low as $1 \mathrm{~ms}$ [8] and the reliability being more than $99.999 \%$ [9]. This will bring stringent requirements to communication system since huge communication resource might be required to secure such extremely high technical specifications. As one of the most effective solutions, packetized predictive control (PPC) conducts robust control under reliable wireless link [10]-[14]. More specifically, a predictive control packet is transmitted from the controller to the plant, and the key is to achieve a good trade-off between real-time control performance and communication resource consumption.

There are two traditional methods of packet transmission that belongs PPC. One is to transmit new packet at every time slot [15] (it is also called continuous transmission). Continuous transmission can guarantee the real-time performance but consume a lot of communication resources (spectrum resources and energy resources). The other is to transmit new packet after the commands in the buffer are empty [16] (it is called intermittent transmission). Intermittent transmission can save a lot of communication resources, but due to the use of many cached predictive control commands, the realtime performance is difficult to achieve. Neither of the method can solve the real-time control performance and communication consumption well at the same time.

In this paper, we consider the control and communication co-design system with short packet wireless communication. We discuss the relationship between the energy efficiency and the real-time performance. In particular, we propose a communication-control co-design method to transmit packet at the optimal time instant to achieve trade-off between the energy efficiency and the realtime performance depending on the application scenarios which can be considered as the general scenarios of the aforementioned two methods. Our results indicate the proposed method could achieve low communication consumption with satisfied real-time performance.

In the rest of this paper, we provide the system model in Section II. In Section III, we first formulate the optimization problem, and then propose a solution to find the optimal transmission time interval. In Section IV, we provide simulation results to verify the effectiveness and advantages of the proposed method. Finally, Section V concludes this paper.

\section{System MOdEL}

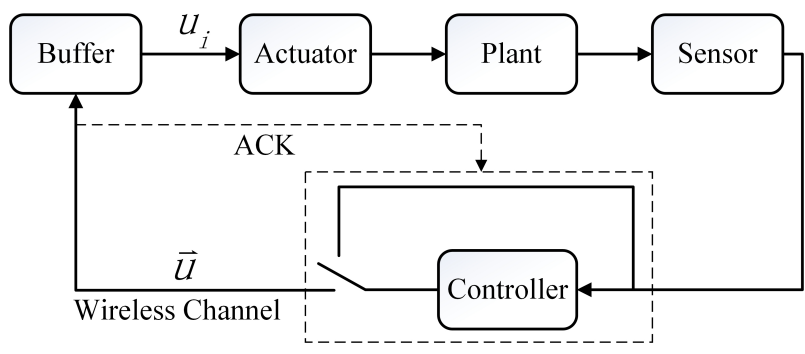

Fig. 1. Wireless PPC Model

In a wireless PPC model provided in Fig. 1, we can observe the working process of a control loop. First, a sensor observes the state of the plant in real time and generates measurements, and then sends these measurements to the controller. Based on these measurements, the controller generates a control command for the current time slot and $r-1$ predicted control commands for future time slots. These control commands $\left(u_{1}, u_{2}, . ., u_{r}\right)$ form a predictive control packet, which is transmitted to the plant by a controller over a wireless channel. If the reception is successful, the current control command $u_{1}$ is sent to an actuator to drive the plant to work and the remaining predictive control commands $u_{2}$ to $u_{r}$ is stored in a buffer. The above working process forms a predictive control loop. 


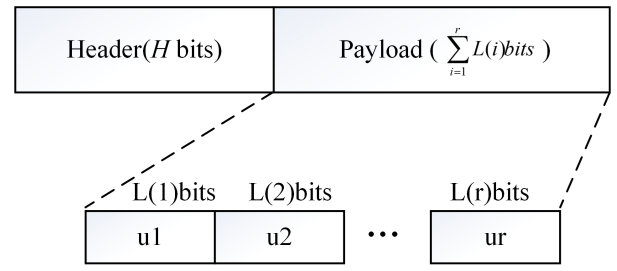

Fig. 2. Packet Model

Since packet $\vec{u}$ contains control commands for $r$ time slots, we have presented a packet model as shown in Fig. 2. Where $H$ is the length of each short packet header, $\sum_{i=1}^{r} L(i)$ is the length of the payload and $L(i)$ is the length of the control command at $i$ th time slot. Then the length of the short packet can be expressed as

$$
N=H+\sum_{i=1}^{r} L(i) .
$$

This paper focuses on the management of packet transmissions from the controller to the plant, with a wireless channel between them as shown in Fig. 1. $A C K$ is the feedback after each transmission. If the packet is successfully received by the plant, then $A C K=1$ is returned to the controller and the predictive control commands are stored in the buffer (overwriting the previously cached commands). If the plant fails to receive the packet, then $A C K=0$ is returned. The controller will manage the next packet transmissions based on the returned $A C K$.

\section{iII. Proposed Packet Transmission Method: COMMUNICATION-CONTROL CO-DESIGN}

In principle, predictive packet transmission in PPC systems should focus on real-time control performance and the communication energy consumption. On the one hand, the real-time control performance depends on how promptly the control commands can be successfully transmitted and used by the actuator. For example, if the actuator executes the first command (real-time control command) in the predictive control packet, the system will have good control performance. On the contrary, if the actuator executes the last command (predicted future control command) in the predictive control packet, the real-time performance will not be as good as the first case. In this paper, we will use information freshness [17], [18] to indicate the real-time control performance. On the other hand, the communication energy consumption depends on the density of transmission. For example, if the transmission is very dense (transmit at every time slot), good real-time performance can be guaranteed, but huge communication energy is consumed. On the contrary, if the transmission is very sparse (transmit after the buffer is empty), little communication energy is consumed, but poor real-time performance may be caused. Therefore, it is reasonable to jointly consider

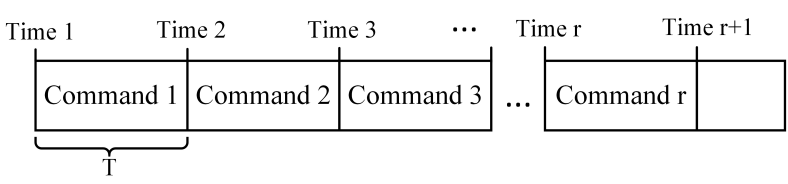

Fig. 3. Transmission Time

the freshness of control commands and communication consumption to manage packet transmission. In this paper, we adopt a method of unifying two objective functions to select the appropriate transmission interval, which can achieve a trade-off between information freshness and energy efficiency.

Next, we first formulate an optimization problem which combines the above two factors in Section II-A, and then find a solution to optimize the problem in section II-B.

\section{A. Problem Formulation}

We first formulate the optimization problem of energy efficiency subject to the control system QoS constraint. Specifically, we will minimize the energy utilization while keep the freshness of the information at the controller.

According to the communication capacity formula for short packets [6]

$$
\begin{aligned}
R\left(n, p_{e}\right) & =C-\sqrt{\frac{V}{n}} Q^{-1}\left(p_{e}\right)+o\left(\frac{\log _{2} n}{n}\right) \\
& \approx C-\sqrt{\frac{V}{n}} Q^{-1}\left(p_{e}\right)+\frac{\log _{2} n}{2 n},
\end{aligned}
$$

where

$$
\begin{gathered}
C=\log _{2}(1+\gamma) \\
=\log _{2}\left(1+\frac{P_{0} G}{N_{0}}\right), \\
V=\gamma \frac{2+\gamma}{(1+\gamma)^{2}}\left(\log _{2} e\right)^{2}, \\
n=B * T .
\end{gathered}
$$

In (2), (3), (4), and (5), $p_{e}$ is the packet loss probability, $\gamma$ is the signal-to-noise ratio, $C$ is the Shannon capacity with unit bandwidth, $N_{0}$ is the power spectral density of the additive white Gaussian noise (AWGN), $G$ is the wireless channel gain (channel fading coefficient). Here, we assume that $G$ is known to the system. $V$ is the channel dispersion coefficient, $n$ is the time-frequency resource used in each packet and $Q^{-1}(\cdot)$ is the inverse Gaussian Q-function. According to [13], $p_{e}$ can be obtained by

$$
p_{e}=Q\left(\frac{n C-N+\frac{\log _{2} n}{2}}{\sqrt{n V}}\right) .
$$

Since a packet contains $r$ control commands for $r$ time slots. Assume that the moment when the plant successfully receives the packet is time 1 , then the time of the next transmission can be selected as time 2 to time $r+1$ as shown in Fig. 3. Choosing to start a new 
transmission at time $k(2 \leq k \leq r+1)$ means that there are $r+2-k$ chances to make the transmission successful. The rationale behind is that if the transmission fails before time $r+1$, the previously cached commands can be used to achieve satisfied control performance. Therefore, the larger the $k$ means less transmission times to ensure successful transmission, and more cached commands are used. Suppose that the system outage probability is $p_{s}$, then the following inequalities should to be satisfied [13].

$$
p_{e}^{r+2-k} \leq p_{s} .
$$

Denote $P_{0}$ as the transmission power spectral density, then we can get the first objective function (energy efficiency $E E[19]$ ) under the condition of satisfying the system outage probability.

$$
\begin{aligned}
& \underset{P_{0}}{\operatorname{Max}}: E E=\frac{N}{(r+2-k) P_{0} n}, \\
& \text { s.t. } \\
& p_{e} \leq p_{s}^{\frac{1}{r+2-k}}, \\
& p_{e}=Q\left(\frac{n C-N+\frac{\log _{2} n}{2}}{\sqrt{n V}}\right) \text {, } \\
& C=\log _{2}(1+\gamma) \text {, } \\
& N=H+\sum_{i=1}^{r} L(i) \text {, } \\
& V=\gamma \frac{2+\gamma}{(1+\gamma)^{2}}\left(\log _{2} e\right)^{2} .
\end{aligned}
$$

As aforementioned, the PPC system includes a current time control command and $r-1$ predicted control commands for the next $r-1$ time slots. Although the control commands predicted by PPC are already very accurate, there are still certain differences compared to real-time transmission of control commands, and the longer the predicted time, the lower the accuracy of its information. As can be seen from Fig. 3, selecting different transmissions at different times will result in different numbers of predicted commands being used. How much of the predicted commands been used will affect the freshness of the information. Therefore, we use the following formula to represent the freshness of the information when it is transmitted at time $k$.

$$
U(k)=1-\sum_{i=3}^{k} \frac{\left\|X_{i-1}(i-1)-X_{i-1}(1)\right\|^{2}}{\left\|X_{i-1}(i-1)\right\|^{2}} .
$$

In (9), $X_{i}(j)(j \leq i)$ represents the control command of time $j$ acquired at time $i$. When $i=j$, it indicates that the command was obtained in real time. The larger $U(k)$ is, the greater the information freshness is. From this we can get another objective function,

$$
\underset{k}{\operatorname{Max}}: U(k)=1-\sum_{i=3}^{k} \frac{\left\|X_{i-1}(i-1)-X_{i-1}(1)\right\|^{2}}{\left\|X_{i-1}(i-1)\right\|^{2}} \text {. }
$$

\section{B. Solution}

1) Optimize transmit power $P_{0}$ based on fixed $k$ :

Since smaller packet-dropout probability $p_{e}$ means higher reliability which must bring more energy cost, we have $p_{e}^{r+2-k}=p_{s}$ when the object function is minimal Thus

$$
p_{s}^{\frac{1}{r+2-k}}-Q\left(\frac{n C-N+\frac{\log _{2} n}{2}}{\sqrt{n V}}\right)=0 .
$$

According to [13], we can obtain the optimal $P_{0}$ at the fixed $k$

$P_{0}(k)=\left(2^{\frac{1}{n}\left(\sqrt{n} Q^{-1}\left(p_{s}^{\frac{1}{r+2-k}}\right) \log _{2}(e)+N-\frac{1}{2} \log _{2}(n)\right)}-1\right) \times \frac{N_{0}}{G}$.

2) Find the optimal $k$ based on information freshness and energy efficiency:

$k$ affects both energy efficiency $E E$ and information freshness $U$. Since the two objective function values are very different in magnitude, it is necessary to first normalize the target function value between $[0,1]$. We use the object function normalization method to unify the two objective functions.

$$
\begin{aligned}
& \max _{k}: F(k)=\frac{E E(k)-E E_{\min }}{E E_{\max }-E E_{\min }}-\sin \left(2 \pi \frac{E E(k)-E E_{\min }}{E E_{\max }-E E_{\min }}\right) \\
& \quad+\frac{U(k)-U_{\min }}{U_{\max }-U_{\min }}-\sin \left(2 \pi \frac{U(k)-U_{\min }}{U_{\max }-U_{\min }}\right), \\
& \text { s.t. } \\
& \quad p_{e} \leq p_{s}^{\frac{1}{r+2-k}} .
\end{aligned}
$$

Since $U$ is only related to $k$, thus it is easy to calculate. In the previous step we have calculated the optimal transmit power $P_{0}$, so $E E$ is also easy to calculate. Therefore, the value of $F(k)$ can be obtained when $k$ takes a different value. Finally, we can get the value of $k$ when $F(k)$ is maximized.

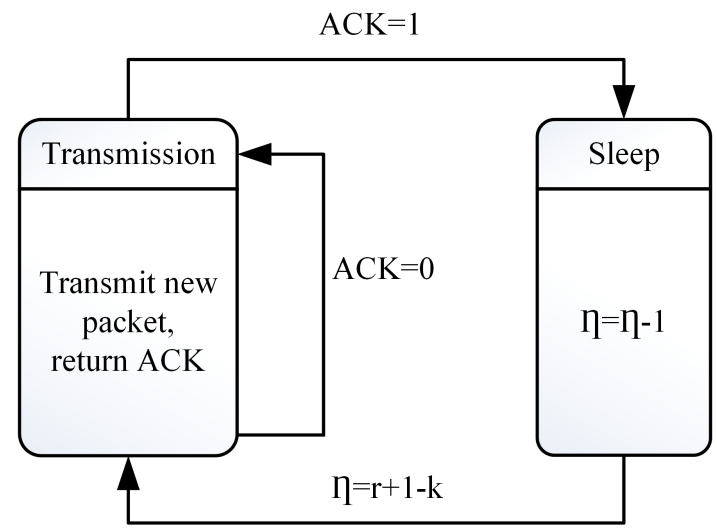

Fig. 4. Controller's modes of operation

When $k$ is determined, we give the operating mode of the controller as shown in Fig. 4. As you can see, the controller operates in two distinct modes: transmission and sleep. In transmission mode, the controller transmits a new predictive control packet to the plant, plant returns an $A C K$ to the controller. The controller adjusts the operating mode based on the returned $A C K$. If $A C K=0$, it indicates that the transmission failed, and the controller continues to transmit new packets. If $A C K=1$, it indicates that the transmission is successful, 

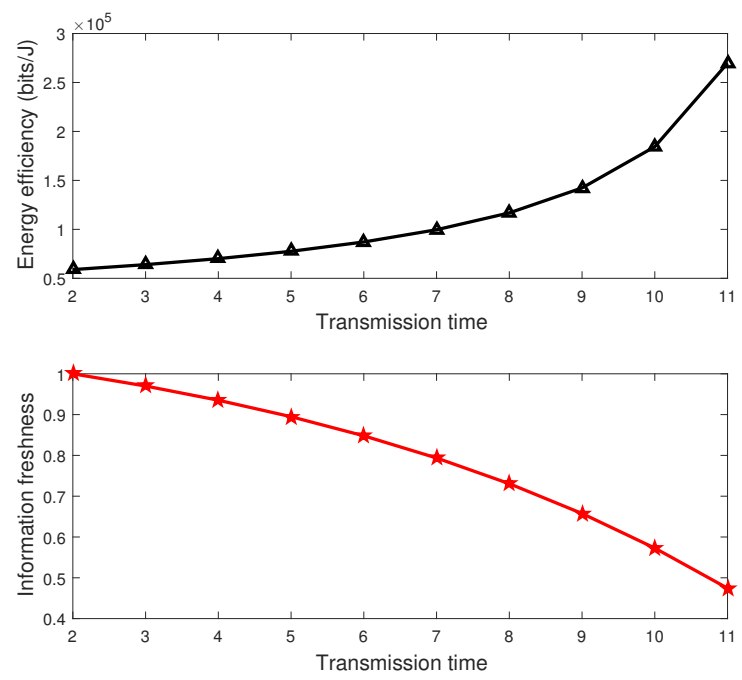

Fig. 5. Effect of transmission time on energy efficiency and information freshness, where packet length is set to 120 .

then the controller moves to sleep mode. In sleep mode, the controller will stop transmitting packets and the plant will use the commands stored in the buffer. Once there are $r+1-k$ remaining commands in the buffer, the controller will be triggered to enter the transmission mode.

\section{Simulation Results}

In this section, we provide the simulation results to demonstrate the performance of the proposed method. The parameter settings are in Tab. I.

TABLE I

\section{PARAMETER SETTINGS}

\begin{tabular}{|c|c|}
\hline Parameters & Values \\
\hline The number of control commands in each packet $: r$ & 10 \\
Wireless channel gain $: G$ & $-113 \mathrm{~dB}$ \\
Time-frequency resources $: n$ & 50 \\
Control outage probability $: p_{s}$ & $10^{-9}$ \\
Noise power density $: N_{0}$ & $-114 \mathrm{dBm}$ \\
\hline
\end{tabular}

In Fig. 5, we compared the effects of different transmission time on both energy efficiency and information freshness. As we can see, larger the transmission interval, the higher the energy efficiency and the lower the information freshness will be achieved. In terms of energy efficiency, larger transmission interval leads to a less number of transmission and thus smaller energy is required to finish the control task. From the freshness perspective, according to equation (9), larger interval leads to a less frequency of transmission and more cached old control commands are used. That is, the bigger the $k$ leads to the smaller the $U(k)$. Therefore, whether it is continuous transmission (transmit new packet at time 2) or intermittent transmission (transmit new packet at time 11) can only achieve one of the highest performance, while the other is the lowest.

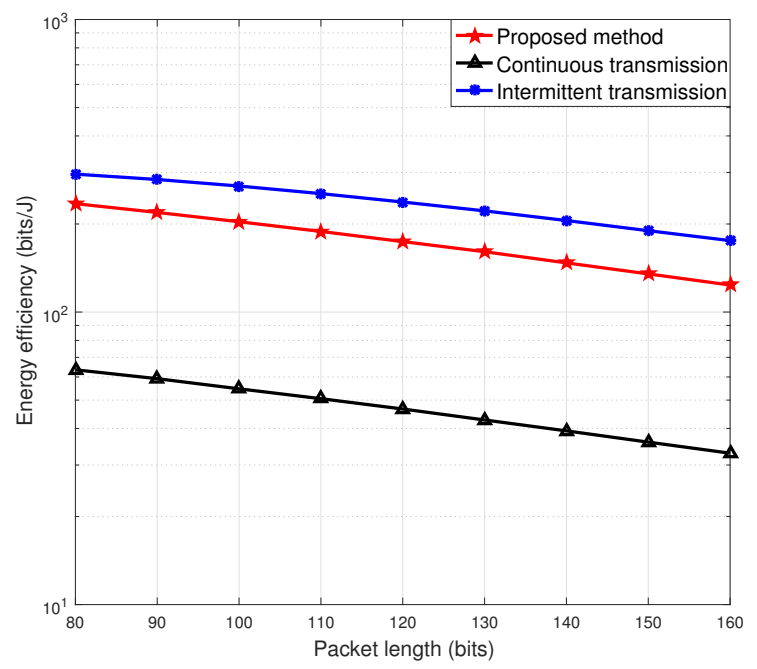

Fig. 6. Energy efficiency versus the packet length.

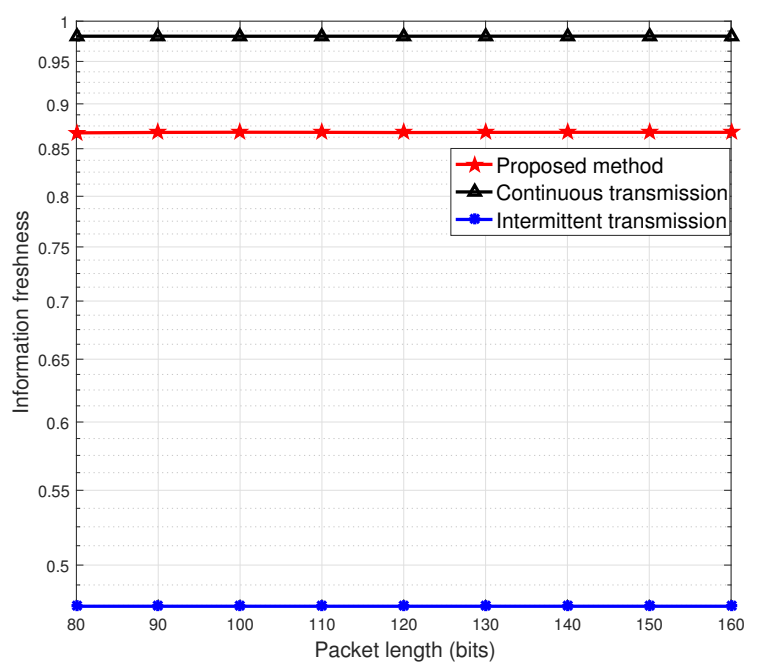

Fig. 7. Information freshness versus the packet length.

In Fig. 6, as the length of the data packet increases, the energy efficiency curves of proposed method, continuous transmission method and intermittent transmission method all decrease. This is because as the length of the data packet increases, higher transmission power is required to ensure the success rate of transmission. Therefore, the energy efficiency decreases. In Fig. 7, as the packet length increases, the information freshness of proposed method, continuous transmission method and intermittent transmission method are flat. This is because that even though the packet length is different but the final transmission success rate is the same since the transmit power is adjusted. In a successfully transmitted data packet, the proportion of control commands at each moment is the same, so the final freshness of information 
does not change with the length of the data packet.

Compared with Fig. 6 and Fig. 7, you can see that the continuous transmission has the highest energy efficiency but the lowest information freshness while the intermittent transmission has the highest information freshness but the lowest energy efficiency. Therefore, the traditional method did not achieve a good trade-off between energy efficiency and information freshness, and both focused on only one performance. In order to make a good trade-off between energy efficiency and information freshness, we propose a method that starts new transmissions at time $k$. As you can see from the figure, our method has similar energy efficiency as the continuous transmission method, and similar information freshness as the intermittent transmission method. Therefore, the proposed method obtains the advantages of the two conventional methods and has good performance in terms of energy efficiency and information freshness.

\section{CONCLUSIONS}

In this paper, we first investigated the effect of the transmission time interval on energy efficiency and information freshness. To achieve good trade-off between information freshness and energy efficiency, we have formulated and solved an optimization problem in terms of the transmission interval. Finally, we verified the performance of the proposed method through simulation, which shows the effectiveness of the proposed algorithms.

\section{REFERENCES}

[1] I. Aktas, M. H. Jafari, J. Ansari, T. Dudda, S. A. Ashraf and J. C. S. Arenas, "LTE evolution - Latency reduction and reliability enhancements for wireless industrial automation," in Proceedings of IEEE 28th Annual International Symposium on Personal, Indoor, and Mobile Radio Communications (PIMRC), pp. 1-7, Oct. 2017.

[2] Z. H. Qing and Z. Wei, "Research on speed detection method of tracked vehicle automatic driving system," in Proceedings of IEEE International Conference on Artificial Intelligence and Big Data (ICAIBD), pp. 256-260, May 2018.

[3] O. Boyko et al., "Multifunctional mobile information system with feedback for analysis of human health and improved access to medical services," in Proceedings of IEEE International Conference on Advanced Information and Communication Technologies (AICT), pp. 168-173, July 2017.

[4] H. Shariatmadari, S. Iraji, R. Jantti, P. Popovski, Z. Li and M. A. Uusitalo, "Fifth-Generation Control Channel Design: Achieving Ultrareliable Low-Latency Communications," IEEE Vehicular Technology Magazine, vol. 13, no. 2, pp. 84-93, June 2018.

[5] T. Hößler, M. Simsek and G. P. Fettweis, "Mission Reliability for URLLC in Wireless Networks," IEEE Communications Letters, Sept. 2018.

[6] G. Durisi, T. Koch and P. Popovski, "Toward Massive, Ultrareliable, and Low-Latency Wireless Communication With Short Packets," in Proceedings of the IEEE, vol. 104, no. 9, pp. 1711-1726, Sept. 2016.

[7] J. J. Nielsen, R. Liu and P. Popovski, "Ultra-Reliable Low Latency Communication Using Interface Diversity," IEEE Transactions on Communications, vol. 66, no. 3, pp. 1322-1334, Mar. 2018.

[8] D. Van Den Berg et al., "Challenges in Haptic Communications Over the Tactile Internet," IEEE Access, vol. 5, pp. 23502-23518, Oct. 2017.

[9] G. P. Fettweis, "The Tactile Internet: Applications and Challenges," IEEE Vehicular Technology Magazine, vol. 9, no. 1, pp. 64-70, Mar. 2014.
[10] Q. Wang, Y. Zou, and Y. Niu, "Event-triggered model predictive control for wireless networked control systems with packet losses," in Proceedings of IEEE International Conference on Cyber Technology in Automation, Control, and Intelligent Systems (CYBER), pp. 1281-1286, June 2015.

[11] J. Huang, M. Lee, M. Tsai, and S. Chiu, "Generalized predictive control in a wireless networked control system," in Proceedings of 9th World Congress on Intelligent Control and Automation (WCICA), pp. 870-875, Oct. 2011.

[12] S. Qin, and T. Badgwell, "A survey of industrial model predictive control technology." Control Engineering Practice, vol. 11, no. 7, pp. 733-764, Nov. 2003.

[13] X. Tong, G. Zhao, M. A. Imran, Z. Pang and Z. Chen, "Minimizing Wireless Resource Consumption for Packetized Predictive Control in Real-Time Cyber Physical Systems," in Proceedings of IEEE International Conference on Communications Workshops (ICC Workshops), pp. 1-6, July 2018.

[14] D. Quevedo, E. Silva, and G. Goodwin, "Packetized predictive control over erasure channels," in Proceedings of American Control Conference (ACC), pp. 1003-1008, July 2007.

[15] B. Demirel, V. Gupta, D. E. Quevedo and M. Johansson, "On the Trade-Off Between Communication and Control Cost in EventTriggered Dead-Beat Control," IEEE Transactions on Automatic Control, vol. 62, no. 6, pp. 2973-2980, June 2017.

[16] D. E. Quevedo and D. Nesic, "Input-to-State Stability of Packetized Predictive Control Over Unreliable Networks Affected by Packet-Dropouts," IEEE Transactions on Automatic Control, vol. 56, no. 2, pp. 370-375, Feb. 2011.

[17] Q. He, D. Yuan, and A. Ephremides, "Optimizing Freshness of Information: On Minimum Age Link Scheduling in Wireless Systems," in Proceedings of 14th International Symposium on Modeling and Optimization in Mobile, Ad Hoc, and Wireless Networks (WiOpt), pp. 115-122, May 2016.

[18] C. Joo, and A. Eryilmaz, "Wireless Scheduling for Information Freshness and Synchrony: Drift-based Design and Heavy-Traffic Analysis," in Proceedings of 15th International Symposium on Modeling and Optimization in Mobile, Ad Hoc, and Wireless Networks (WiOpt), pp. 1-8, May 2017.

[19] F. Mriaux, S. Valentin, S. Lasaulce and M. Kieffer, "An energyefficient power allocation game with selfish channel state reporting in cellular networks," in Proceedings of 6th International ICST Conference on Performance Evaluation Methodologies and Tools, pp. 290-297, Dec. 2012. 